Slavica

bruxellensia

\section{Slavica bruxellensia}

Revue polyphonique de littérature, culture et histoire

slaves

$10 \mid 2014$

Espace slave, espace germanique

\title{
Edito \#10
}

Dorota Walczak

\section{OpenEdition}

Journals

Édition électronique

URL : http://journals.openedition.org/slavica/1669

DOI : 10.4000/slavica. 1669

ISSN : 2034-6395

Éditeur

Université libre de Bruxelles - ULB

\section{Référence électronique}

Dorota Walczak, «Edito \#10 », Slavica bruxellensia [En ligne], 10 | 2014, mis en ligne le 15 avril 2014 consulté le 22 septembre 2020. URL : http://journals.openedition.org/slavica/1669 ; DOI : https:// doi.org/10.4000/slavica.1669

Ce document a été généré automatiquement le 22 septembre 2020.

\section{(c) (i) () $\Theta$}

Les contenus de Slavica bruxellensia sont mis à disposition selon les termes de la Licence Creative Commons Attribution - Pas d'Utilisation Commerciale - Pas de Modification 3.0 France. 


\section{Edito \#10}

\section{Dorota Walczak}

1 Dans son étonnant poème Pan Kichot (1960), Günter Grass, prix Nobel de Littérature et personnalité hors du commun, aussi controversé que génial, fait la démonstration d'une extraordinaire habilité à gérer le littéraire et le réel. Avec une vraie maestria, il mélange l'élément lyrique et épique, l'intertextuel et le pictural, l'historique et le fabuleux, l'individuel et le collectif d'une manière puissante, évidente mais aussi sibylline. La part de mystère se cache dans la réinscription de son nouveau Don Quichotte - le hussard polonais qui avec sa lance attaque les moutons - la division blindée des tanks. En invitant ainsi le personnage de Don Quichotte à siéger dans son poème, Grass nous invite, en tant que lecteurs de Cervantès, à revisiter la vivacité des modèles littéraires ainsi qu'à les confronter à l'histoire: ici la Deuxième Guerre mondiale. L'auteur de Die Blechtrommel (Le Tambour, 1959) nous suggère à notre tour d'éplucher comme un artichaut les significations des mots et des vers qui dévoilent des images stéréotypées et pourtant toujours actives et prêtes aux confrontations de la vérité littéraire avec la vérité historique et sociétale. Au centre de ce poème se situe la confrontation $d u$ caractère polonais et du caractère allemand, du "slave » et du "germanique », il s'agit bien du souci d'évoquer des clichés imagés et de les remettre en question. C'est aussi la vraie préoccupation des auteurs qui contribuent au dixième numéro de Slavica Bruxellensia : réexaminer des images forgées dans l'histoire et dans la littérature européennes dans leurs domaines de recherche respectifs. Ainsi, l'article de Kornelia Ćwiklak nous montre un vaste panorama fort utile de liens réciproques et complexes dans la prose polonaise et allemande d'après 1945, tandis qu'Igor Fiatti examine la notion de déplacement et des frontières dans l'œuvre de Peter Handke. Eloïse Adde-Vomáčka revient aux sources même de l'image que les Tchèques ont des Allemands dans l'une des plus précieuses chroniques slaves du XIV siècle, la Staročeská kronika tak řěceného Dalimila (Chronique de Dalimil). Nous présentons également l'article de Valentina Toujikova qui se penche sur la problématique des emprunts de l'allemand dans la langue russe et celui d'Alexia Gassin sur les contacts russo-allemands dans l'expressionisme cinématographique de Berlin. Dans notre rubrique Excellensia Hubert Roland et Svetlana Cecovic commentent l'œuvre de Benjamin Goriély. Nous nous réjouissons également de pouvoir publier les prémices de la nouvelle traduction de 
Sklepy cynamonowe (Les Boutiques de Cannellei) de Bruno Schulz réalisée par Alain Van Crugten et présenter la personnalité et les recherches de Pieter Lagrou dans un entretien mené par Petra James et Cécile Bocianowski. Ce numéro se termine comme de coutume par des recensions d'ouvrages récents.

2 Bonne lecture à tous, merci à toute l'équipe qui a contribué à préparer ce numéro et à bientôt pour le prochain numéro consacré à la rencontre de la philosophie et de la littérature slaves.

\section{AUTEUR}

\section{DOROTA WALCZAK}

Responsable de la Chaire de Polonais, section de Langues et Littératures modernes, option Slaves, de l’Université Libre de Bruxelles (Belgique) ; Rédactrice en chef de Slavica Bruxellensia 